\title{
ПОНЯТИЙНЫЕ СТРУКТУРЫ ЗНАНИЙ И КОГНИТИВНЫЙ СТИЛЬ
}

\author{
Т.А. ГАВРИЛОВА
}

${ }^{a}$ Высшая школа менеджмента СПбГУ, 199004, Россия, Санкт-Петербург, Волховский пер., д. 3

\begin{abstract}
Резюме
Структурирование знаний - один из малоизученных когнитивных процессов, который существенно влияет как на процессы обучения, так и на эффективность процессов передачи знаний в рамках профессиональной деятельности. В статье представлены результаты исследования влияния индивидуальных характеристик когнитивного стиля на процессы структурирования информации при обучении. Акцент ставится на изучение тех характеристик когнитивного стиля, которые значимо влияют на особенности построения концептуальных иерархических моделей (так называемых онтологий), важных для усвоения и понимания материала. Описаны результаты эмпирической проверки гипотез о влиянии таких характеристик индивидуального когнитивного стиля эксперта, как полезависимость/поленезависимость, узость/широта категории и импульсивность/рефлективность, на особенности индивидуального и коллективного формирования онтологий. Особый интерес представляют исследования групповой работы (в парах и тройках) по созданию единой структуры предметной области и их связи с индивидуальными представлениями о предмете членов группы. В качестве испытуемых выступали студенты СанктПетербургского политехнического университета, которые сначала были протестированы на различия в когнитивных стилях, а затем им было предложено создать онтологии понятия «информатика» как индивидуально, так и в группах. Полученные результаты позволяют предложить ряд рекомендаций по формированию групп для успешной коллективной работы, в частности, разработки баз данных, моделей представления знаний, бизнес-моделей и других концептуальных структур.
\end{abstract}

Ключевые слова: понятийные структуры, онтологии, онтологический инжиниринг, структурирование знаний, категоризация, когнитивные стили.

\section{Введение}

В условиях жестких информационных перегрузок, мультизадачности и мультимодальности учебных процессов в образовательных орга- низациях всех профилей наиболее острой становится проблема компрессии и структурирования учебной информации для повышения эффективности преподавания. Статья посвящена актуальной проблеме

Исследование начато в рамках проекта РФФИ (проект № 11-07-00140а) и завершено при поддержке РНФ (проект № №15-18-30048). 
использования моделей представления знаний в форме онтологий (упрощенно «концептуальных моделей») для обучения и контроля знаний студентов. Акцент сделан на когнитивных аспектах.

По прогнозам исследования компании IDC (IDC, 2014) объемы информации будут удваиваться каждые два года в течение следующих восьми лет. Таким образом, усложняются процессы структурирования информации для ее понимания, хранения и передачи. В работе Л.Л. Любимова (2007), в частности, подчеркивается, что на фоне постоянно нарастающего объема информации умение учителя «пропустить» ее через себя и представить в сжатом виде, удобном для изучения, выходит на первый план. При этом важно, чтобы ученики усваивали не столько отдельные фрагменты информации, сколько взаимосвязи между понятиями и структуру предмета в целом. В вузах этот феномен отличается высоким уровнем сложности предметных областей.

Переход от текста и таблиц к «простым» картинкам-структурам можно считать одним из первых эффективных способов компрессии знаний. Такие понятийные структуры могут служить каркасом или структурированным словарем, обеспечивающим понимание предмета. Их можно использовать для коллективных упражнений, они упрощают коммуникации, определяя контекст, в рамках которого работает группа.

C точки зрения обучения это важно не только для учеников, но и для преподавателей. В высшей школе нередки ситуации, когда одна дисциплина ведется несколькими пре- подавателями, каждый из которых имеет свое собственное представление о предмете. Описание программы дисциплины на языке понятийных структур помогает точнее определить рамки изучаемого предмета, а также позволяет избежать различного толкования понятий преподавателями (Гаврилова и др., 2011).

При структурировании знаний на первом шаге выявляются наиболее важные сущности (или концепты) предметной области, на втором шаге они объединяются в категории, а затем выделяются взаимосвязи между этими сущностями и категориями. Категоризация в широком смысле это процесс образования и выделения самих категорий, членения внешнего и внутреннего мира человека, упорядоченное представление разнообразных явлений через сведе́ ние их к меньшему числу разрядов или объединений, а также результат классификационной (таксономической) деятельности (Кубрякова и др., 1997). В работах ведущих российских и зарубежных исследователей в области когнитивных процессов (Аллахвердов, 2006; Величковский, 2006; Соловьев, 2007; Солсо, 2006; Холодная, 2009; Chernigovskaya, 1999; Lakoff, 1987; Rosch, 1999; и мн. др.) неизменно подчеркивается сложность и неоднозначность процессов категоризации понятий.

В данной работе процесс категоризации (или формирования понятийных структур) рассматривается на примере визуального построения онтологий - концептуальных моделей предметных областей.

В качестве испытуемых выступали студенты политехнического университета, которые сначала были 
протестированы на различия в когнитивных стилях, а затем им было предложено создать онтологии понятия «информатика» как индивидуально, так и в группах.

Когнитивные стили - это индивидуальные способы переработки информации о мире в виде различий в восприятии, анализе, структурировании, категоризации и оценивании происходящего (Холодная, 2004).

В статье представлены результаты исследования процессов структурирования и категоризации и выявления индивидуальных характеристик когнитивного стиля, которые значимо влияют на эти процессы. Исследование связей между когнитивными стилями обучающихся и темпом усвоения знаний уже проводились (например: Афанасьев, Резаков, 2012), но в данной работе впервые сделан акцент именно на процессах структурирования. Особый интерес представляют результаты исследования групповой работы (в парах и тройках) по созданию единой структуры некоторой предметной области и их связь с индивидуальными представлениями о предмете членов группы.

\section{Онтологии как способ структурирования знаний}

\section{Основные определения}

Существует несколько подходов к определению понятия «онтология». Одно из самых известных определений дал Том Грубер: «Онтология это спецификация концептуализации» (Gruber, 1993, р. 199). Никола Гуарино определяет онтологию следующим образом: «Онтология - это формальная теория, ограничивающая возможные концептуализации мира» (Guarino, Giaretta, 1995, p. 30). B обеих формулировках используется понятие «концептуализация», требующее, в свою очередь, определения. Приведем более развернутое определение: «Онтология - это спецификация предметной области или формальное ее представление, которое включает словарь указателей на термины предметной области и логические выражения, которые описывают, что́ эти термины означают, как соотносятся друг с другом и как они могут или не могут быть связаны между собой» (Гаврилова, Муромцев, 2007, с. 320).

В общем виде структура онтологии представляет собой следующий набор элементов: понятия, отношения, аксиомы, отдельные экземпляры. Понятия рассматриваются как концептуализации класса всех представителей некой сущности или явления. Классы (или понятия) являются общими категориями, которые могут быть упорядочены иерархически. Каждый класс описывает группу индивидуальных сущностей, которые объединены на основании наличия общих свойств. Понятия могут быть соединены различного рода отношениями, которые связывают классы и описывают их. Самым распространенным типом отношений, использующимся во всех онтологиях, является отношение категоризации, т.е. отнесение к определенной категории.

Аксиомы задают условия соотнесения категорий и отношений, они выражают очевидные утверждения, связывающие понятия и отношения. Наряду с указанными элементами 
онтологии в нее также входят так называемые экземпляры. Экземпляры - это отдельные представители класса сущностей, или явлений, т.е. конкретные элементы какой-либо категории.

Все более широкое применение онтологии находят в «мягких» науках - менеджменте, экономике, электронном бизнесе, где структурирование информации представляет значительную сложность. Применяются онтологии и в преподавании различных дисциплин наряду с другими наглядными представлениями, такими как диаграммы, схемы, графики, планы и т.п. Необходимо отметить, что онтологии часто субъективны, так как часть знаний зависит от опыта и предпочтений субъекта. Использование «чужих» онтологий - это удобный и компактный способ получения новых знаний.

Таким образом, онтология как понятийная структура описывает иерархическую систему терминов, являясь структурированным словарем предметной области.

В качестве инструмента визуализации онтологий (Гаврилова и др., 2011) можно использовать интеллект-карты, или mind-maps (Бьюзен, Бьюзен, 2014). Рисунок 1 иллюстрирует фрагмент классификации онтологий в форме интеллект-карты.

\section{Оценка онтологий}

Вопрос оценки качества онтологий остается открытым, так как существуют различные критерии оценки. Авторы данной статьи считают, что во многих случаях можно оценивать качество онтологии с эргономических позиций, или с позиции ясности визуального представления. Для этого выделяют ряд характеристик, которые описывают качество этого представления и часть которых можно измерить. Для выделения

Классификация онтологий

Рисунок 1

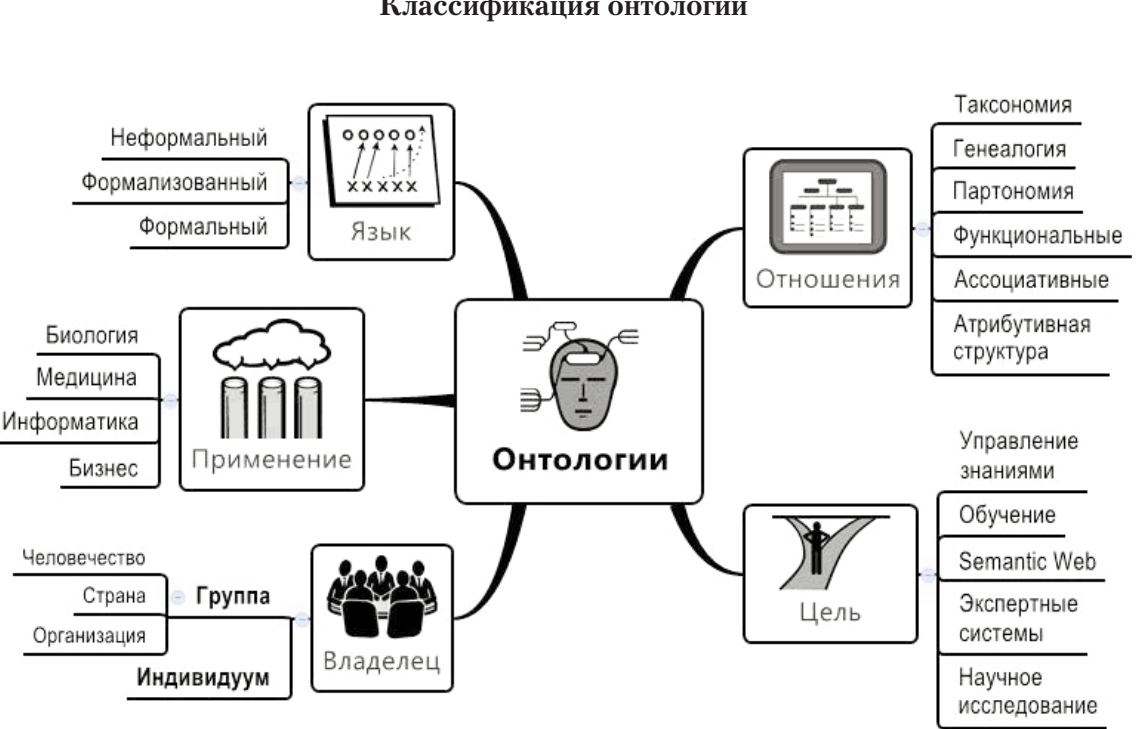


таких характеристик использовались «нечеткие», но интуитивно понятные правила «хорошей» онтологии, сформулированные Гавриловой (2002, 2005).

- Понятия одного уровня иерархии связываются с родительским концептом одним и тем же типом отношения (например, «класс - подкласс» или «часть - целое»).

- Глубина ветвей онтологического дерева должна быть примерно одинаковой $( \pm 2)$.

- Общий вид онтологии должен быть довольно симметричным.

- Перекрестные ссылки должны быть по возможности исключены.

- Максимальное число концептов одного уровня, или глубина ветви, не должна превышать извест-

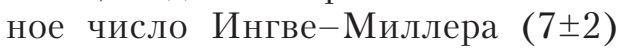
(Miller, 1956).

Оценивать качество построенных онтологий можно двумя способами:

- Экспертный - аналитик-онтологист и эксперты предметной области оценивают качество по различным критериям, таким как полнота, сбалансированность, адекватность и т.П.

- Формализованный - с помощью набора метрик, по которым оценивается каждая онтология.

Второй способ предпочтительнее по таким причинам, как отсутствие субъективизма и возможность автоматизации. Большой вклад в методы формализованной оценки внес А. Гангеми (Gangemi et al., 2006).

Для оценки онтологий использовался расширенный набор метрик (Болотникова и др., 2011), часть которых описана ниже.

При описании метрик использованы следующие обозначения: $g$ - граф, представляющий онтологию, концепты (классы и экземпляры) онтологии являются вершинами графа, отношения между концептами представлены в виде ребер графа;

$G$ - множество всех вершин графа $g$;

$L E A \subseteq G-$ множество листьев графa $g$;

$S I B_{v \in G}-$ множество вершин графа $g$ (называемое семейством), для которых вершина $\nu$ является непосредственным суперклассом;

$E-$ множество всех ребер $g$.

\section{Метрики глубинь}

Абсолютная глубина вычисляется как сумма длин всех путей графа (где путем называется любая последовательность соединенных между собой вершин, начинающаяся от корневой вершины и заканчивающаяся листом графа):

$$
m=\sum_{j}^{P} N_{j \in P},
$$

где $j \in P-$ длина каждого пути $j$ из множества путей $P$ графа $g$.

\section{Минимальная глубина:}

$$
M=N_{j \in P}, \quad \forall i\left(N_{j \in P} \leqslant N_{i \in P}\right),
$$

где $N_{j \in P}$ и $N_{i \in P}-$ длины пути $j$ и $i$ из множества путей $P$ графа $g$.

\section{0-я процентиль глубины:}

$$
m=P_{90}\left(N_{j \in P}\right),
$$

где $P_{90}\left(N_{j \in P}\right)-90$-я процентиль глубины графа (возможное значение длины пути графа, которое не превышают длины 90\% путей графа). 
Метрики шириньь

Абсолютная ширина:

$$
m=\sum_{j}^{L} N_{j \in L},
$$

где $N_{j \in L}-$ количество вершин на уровне $j$ из множества уровней $L$ графа $g$.

Средняя ширина вычисляется как абсолютная ширина, деленная на количество уровней иерархии:

$$
m=\frac{1}{n_{L \subseteq g}} \sum_{j}^{L} N_{j \in L}
$$

где $N_{j \in L}-$ количество вершин на уровне $j$ из множества уровней $L$ графа $g, n_{L \subseteq g}-$ количество всех уровней графа (максимальная глубина графа, увеличенная на 1, если рассматривать только выбранное доминирующее отношение).

Среднее квадратичное отклонение отношения ширины соседних уровней:

$$
m=\frac{\sum_{i=2}^{n_{L I g}}\left(\frac{N_{l_{i \in L}}}{N_{l_{l-1} \in L}}-\frac{1}{n_{L \subseteq g}-1} \sum_{i=2}^{n_{L g g}} \frac{N_{l_{i \in E L}}}{N_{l_{l-1} \in L}}\right)^{2}}{n_{L \subseteq g}-1} .
$$

\section{Метрики запутанности}

Запутанность онтологии определяется как количество вершин, у которых есть несколько непосредственных суперклассов, деленное на количество вершин графа онтологии. Таким образом, в онтологиях, где нет множественного наследования, эта метрика будет равняться нулю. Чем чаще множественное наследование используется в онтологии, тем хуже она с точки зрения когнитивной эргономики.
Среднее количество родительских вершин у вершины графа:

$$
m=\frac{1}{n_{G}} \sum_{v}^{G} N_{S_{v \in G}}
$$

где $S_{v}=\{a \in G \mid i s a(\nu, a)\}$ - множество всех родителей вершины $\nu$, $N_{S_{v \in G}}$ - количество всех родительских вершин вершины $\nu$,

$n_{G}-$ количество всех вершин графа $g$.

\section{Метрики ветвистости}

(Gangemi et al., 2005)

\section{Абсолютная мощность семейств:}

$$
m=\sum_{j}^{S I B} N_{j \in S I B},
$$

где $N_{j \in S I B}-$ количество вершин в семействе $j$ из множества всех семейств графа $g$.

Коэффициент ветвистости семейств:

$$
m=\frac{1}{n_{G}} \sum_{j}^{S I B} N_{j \in S I B},
$$

где $N_{j \in S I B}-$ количество вершин в семействе $j$ из множества всех семейств графа $g$, $n_{G}$ - количество всех вершин графа .

Абсолютная мощность листьев:

$$
m=n_{L E A \subseteq g},
$$

где $n_{\text {LEA g }}-$ количество листьев графа $g$.

Взвешенный коэффициент ветвистости листьев вычисляется как абсолютная мощность листьев, деленная на абсолютную глубину графа:

$$
m=\frac{n_{L E A \subseteq g}}{\sum_{j}^{P} N_{j \in P}},
$$

где $n_{\text {LEA }}-$ количество листьев графа g, 
$N_{j \in P}$ - длина каждого пути $j$ из множества путей $P$ графа g.

На рисунках 2 и 3 показаны примеры различных онтологических структур.

Для автоматической оценки онтологий и обработки данных использовался программный инструмент COAT-cog, созданный Е.С. Болотниковой (Гаврилова и др., 2009) и вычисляющий метрики следующих категорий:

- метрики циклов,

- метрики Ингве-Миллера,

- метрики разнообразия количества связей,

- метрики разнообразия типов связей концептов,

- метрики глубины,

- метрики ширины,

- метрики запутанности (tangledness) графа,

- метрики измерения ветвистости (fan-outness) графа.

Кроме непосредственного вычисления метрик инструмент СОАТ-cog позволяет получить информацию об этих метриках, их назначении и трактовке значений из встроенного в инструменте словаря. Также на вы- ходе СОАТ выдает список «проблемных» узлов с указанием причины, по которой концепт попал в этот список, например, если вычисленная «степень» узла (количество ребер графа) превышает число ИнгвеМиллера.

\section{Когнитивные стили}

Существует множество факторов, влияющих на процессы категоризации понятий и образование абстрактных классов. Особенности когнитивного стиля, безусловно, также относятся к таким факторам.

Необходимо отметить некоторые важные свойства, присущие когнитивному стилю (КС) (переработано из: Холодная, 2004):

1) КС - это предпочтение определенного способа интеллектуального поведения (т.е. субъект в принципе может выбрать любой способ переработки информации, однако он непроизвольно предпочитает определенный способ восприятия и анализа происходящего, в наибольшей мере соответствующий его психологическим возможностям);
Пример структуры «узкой» и «глубокой» онтологии

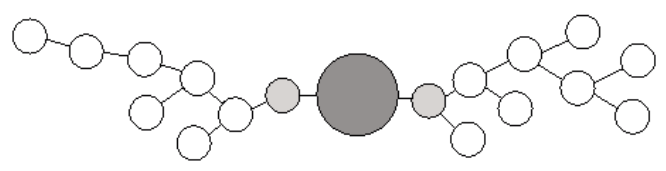

Пример структуры «широкой» онтологии

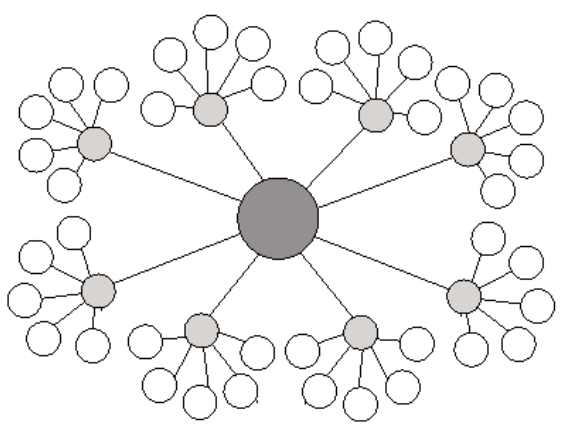


2) КС - это устойчивая характеристика субъекта, стабильно проявляющаяся для разных задач и в разных ситуациях;

3) КС - это биполярное измерение, в рамках которого каждый когнитивный стиль описывается за счет обращения к двум крайним формам интеллектуального поведения (например, в виде импульсивности/ рефлективности и т.д.);

4) к КС неприменимы оценочные суждения, так как представители разных полюсов когнитивного стиля имеют свои преимущества в ситуациях, требующих эффективной индивидуальной адаптации.

В научной литературе по когнитивной психологии можно встретить более 20 психологических характеристик, которые относят к КС. Обычно выделяют следующие характеристики КС (Холодная, 2004):

- полезависимость/поленезависимость;

• узкий/широкий диапазон эквивалентности;

- узость/широта категории;

- ригидный/познавательный контроль;

- толерантность/нетолерантность к нереалистичному опыту;

- фокусирующий/сканирующий контроль;

- сглаживание/заострение;

- импульсивность/рефлективность;

- конкретная/абстрактная концептуализация;

- когнитивная простота/сложность. На основе таких критериев, как значимое влияние на построение иерархических моделей, наличие изученных методик и простота реализации процесса тестирования (в частности, наличие и доступность соответствующего инструментария), для исследования были выбраны три характеристики когнитивного стиля: полезависимость/поленезависимость (ПЗ/ПНЗ), узость/широта категории и импульсивность/рефлективность. В дальнейшем предполагается продолжить исследование, включив в него характеристику «узкий/широкий диапазон эквивалентности» (Косихин, 2012).

\section{Полезависимость/ поленезависимость}

ПЗ/ПНЗ описывает способность восприятия к выделению отдельных объектов из общего контекстного поля, иными словами, выделению сигнала на фоне помех; соответственно, полезависимые индивидуумы склонны рассматривать отдельные объекты и явления в общем контексте, в то время как поленезависимые более склонны абстрагироваться от контекста.

По определению Г. Уиткина (Witkin et al., 1977), П3/ПНЗ - это «структурирующая способность восприятия». Таким образом, характеристика этого стиля влияет на процесс структурирования в целом (т.е. в исследуемом случае на построение онтологий «с нуля»), но в большей степени - на реструктуризацию (при объединении онтологий). ПЗ/ ПНЗ оказывает существенное влияние на процесс решения задач в условиях совместной деятельности. При работе в парах, члены которых имели различные когнитивные стили (ПЗ/ ПНЗ), окончательное решение, как правило, ближе к варианту, предложенному ПНЗ партнером. ПНЗ пары испытывают трудности в выработке 
общего решения по спорным вопросам, тогда как ПЗ испытуемые успешно договариваются между собой при совместном решении проблем (Холодная, 2004).

В качестве методики диагностики полезависимости/поленезависимости испытуемого применялась одна из модификаций методики Л.Л. Терстоуна «Скрытые фигуры» (Thurstone, 1944), представляющей разновидность тестов на визуальное восприятие (Witkin, 1971). Существует несколько модификаций этой методики, но задача испытуемого в любой из них - найти простую фигуру внутри сложной.

Испытуемые проходили тестирование с помощью системы диагностики «Студент» (Котова и др., 2013)

Пример фрагмента теста представлен на рисунке 4 .

Тест содержал 49 подобных заданий. На тест отводилось 10 минут. Перед началом теста испытуемому предъявлялась инструкция по поиску искомой фигуры в одном из четырех рисунков справа. После прохождения теста испытуемый получал оценку его уровня полезависимости/поленезависимости, а в базе данных сохранялись число пройден- ных заданий и количество допущенных ошибок.

\section{Импульсивность/рефлективность}

Различие между полюсами импульсивности и рефлективности заключается в объеме информации, которую индивидуум собирает до принятия решения: импульсивные лица принимают решения на недостаточной информационной основе, тогда как рефлективные - склонны принимать решения с учетом максимально полной информации о ситуации.

Для диагностики импульсивности/рефлективности использовалась методика «Сравнение похожих рисунков» (Kagan, 1966).

Испытуемому предъявляется одно тренировочное задание, а затем 12 основных. В каждом задании было необходимо из восьми (в тренировочном задании - из шести) изображений выбрать одно, полностью совпадающее с эталонным.

Пример пробного задания представлен на рисунке 5 .

В качестве показателей импульсивности/рефлективности в базу данных для последующей обработки

Пример фрагмента теста «Скрытые фигуры».

Рисунок 4 Необходимо определить, в какие из четырех правых рисунков входит искомая фигура, изображенная слева
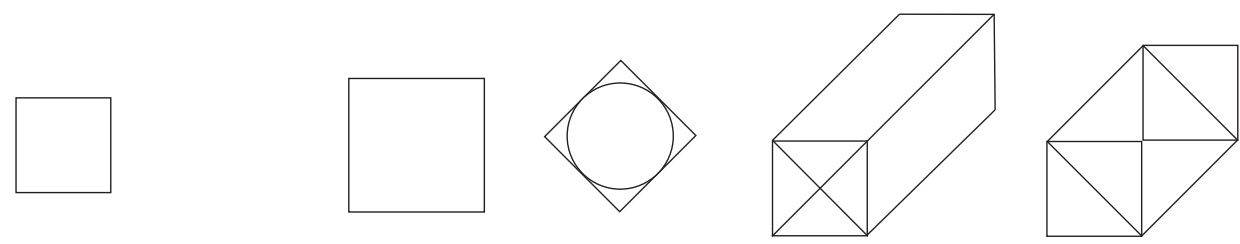
Пример пробного задания теста на импульсивность/рефлективность

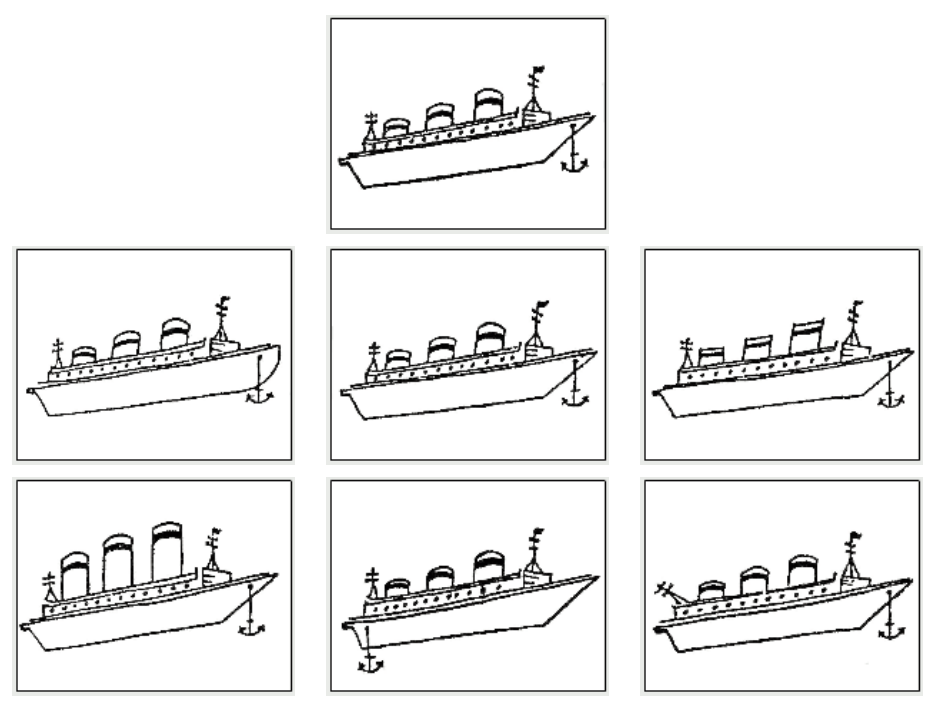

заносились общее количество ошибок и время первого ответа.

\section{Узость/иирота категории}

Характеристика «узость/широта категории» отражает широту осуществляемой индивидуумом категоризации отдельных объектов: «узкие» категоризаторы склонны ограничивать область применения определенной категории, тогда как «широкие» категоризаторы, напротив, склонны подводить под одну категорию большое число подтверждающих ее примеров.

В качестве методики диагностики узости/широты категории применялась одна из модификаций методики «Средние суждения». В проекте использовался вариант методики, предложенный С. Филленбаумом (Fillenbaum, 1959), который состоит в следующем: испытуемый должен указать максимальное, среднее и минимальное значение какого-либо объекта или явления.

Тест был реализован средствами Google docs. Испытуемые отвечали на однотипные вопросы, например:

«Сколько времени (в часах) необходимо для доставки корреспонденции из Москвы в Санкт-Петербург?

- самому быстрому почтовому голубю:

- самому медленному почтовому голубю:

- среднее время доставки: 》.

Показатель широты категории разность максимального и минимального значений (чем она меньше, тем у́же категоризация). Среднее значение использовалось для нормировки.

\section{Описание эксперимента}

Целью представленного исследования являлось изучение влияния индивидуальных когнитивных стилей 
на формирование категорий и создание онтологий, а также исследование процессов групповой разработки онтологий с учетом специфики индивидуальных когнитивных стилей.

Исследование выполнялось на выборке из 79 студентов IV-V курса факультета технической кибернетики Санкт-Петербургского государственного политехнического университета, изучающих курс по разработке интеллектуальных систем. Эксперимент состоял из двух фаз: «индивидуальной» и «коллективной».

Целью «индивидуальной» фазы было исследование взаимосвязей индивидуального когнитивного стиля и особенностей формирования онтологий предметной области. «Индивидуальная» фаза была разбита на четыре этапа.

- Определение когнитивных стилей испьтуемых (Лещева, Котова, 2012): студенты дистанционно в режиме онлайн были протестированы по трем тестам, измеряющим перечисленные выше особенности когнитивного стиля.

- Построение испьтуемыми онтологий для предметной области «информатика» с использованием системы Protégé. Protégé - это свободно распространяемый редактор онтологий для построения баз знаний, созданный в Стэнфордском университете. В качестве методологической основы построения онтологий студенты использовали перевод статьи Н. Ф. Ной и Д.Л. МакГиннесс (Noy, McGuinness, 2001).

- Формализованная оценка онтологий, построенных испытуемыми, путем вычисления метрик в про- граммной среде СОАТ (Гаврилова и др., 2009).

- Статистический анализ полученных данных для определения связи между характеристиками построенных онтологий и когнитивными стилями испытуемых.

«Коллективная» фаза опиралась на результаты, полученные в рамках «индивидуальной» фазы, и состояла в исследовании разработки онтологий в группах путем экспертного анализа объединения испытуемыми построенных ранее онтологий.

\section{«Индивидуальная» фаза}

На основании анализа литературы и эмпирического опыта в разработке онтологий были выдвинуты следующие гипотезы.

- Гипотеза 1: люди, принадлежащие к полюсу ПНЗ, имеют более развитые когнитивно-структурирующие способности, соответственно, качество онтологий, построенных поленезависимыми испытуемыми, будет выше.

- Гипотеза 2: импульсивные индивидуумы строят поверхностные онтологии без достаточной категоризации на верхнем уровне, в то время как рефлективные строят более глубокие онтологии.

- Гипотеза 3: чем больше ошибок совершают испытуемые в тесте Дж. Кагана на «импульсивность/ рефлективность», тем более «запутанные» онтологии они строят.

- Гипотеза 4: когнитивный стиль «узость/широта категорий» влияет на ширину онтологий, т.е. «широкие» категоризаторы склонны строить «широкие» онтологии. 
Обработка полученных результатов была проведена путем анализа и сопоставления метрик онтологий и характеристик когнитивного стиля. При этом использовались возможности программного пакета MS Excel и пакета математической статистики SPSS.

В таблице 1 приведен фрагмент сводной таблицы по двум сериям исследований, в котором указана величина коэффициентов корреляции некоторых метрик с основными показателями двух когнитивных стилей: И/Р - импульсивность/ рефлективность и УК/ШК узость/широта категории (см. таблицу 1). Значимая корреляция метрик с показателем стиля ПЗ/ПНЗ - полезависимость/поленезависимость отсутствует, поэтому в таблице не приводится. Пустая клетка в таблице означает отсутствие значимой корреляции.

Гипотеза 1 не подтвердилась: связи между показателем ПЗ/ПНЗ и рангом онтологии не наблюдается.

Гипотеза 2 частично подтвердилась: метрика «90-я процентиль глубины» коррелирует с временем первого ответа в тесте Дж. Кагана, т.е. рефлективные испытуемые строят более глубокие онтологии. При этом обратной корреляции между временем первого ответа и шириной онтологий не выявлено.

Таблица 1

Значимые коэффициенты корреляции по Спирмену между метриками и показателями когнитивных стилей

\begin{tabular}{|l|c|c|c|}
\hline \multirow{2}{*}{\multicolumn{1}{|c|}{ Метрики }} & \multicolumn{2}{|c|}{ Результаты по методикам } \\
\cline { 2 - 4 } & Время ответа & $\begin{array}{c}\text { Количество } \\
\text { ошибок }\end{array}$ & Размер категории \\
\cline { 2 - 4 } & & & $0.44^{*}$ \\
\hline Количество классов & & & $0.46^{*}$ \\
\hline Количество листьев & & & $0.39^{*}$ \\
\hline Абсолютная глубина & $0.34^{*}$ & & \\
\hline Минимальная глубина & & & $0.54^{*}$ \\
\hline 90-я процентиль глубины & & $0.48^{*}$ \\
\hline Средняя ширина & & $0.47^{*}$ & \\
\hline СКО относительной ширины & & $0.44^{* *}$ \\
\hline Среднее число родителей в узлах & & $0.50^{* *}$ & \\
\hline Абсолютная мощность семейств & & & $0.46^{* *}$ \\
\hline Коэффициент ветвистости семейств & & $-0.39^{* *}$ & \\
\hline Абсолютная мощность листьев & & & \\
\hline $\begin{array}{l}\text { Взвешенный коэффициент } \\
\text { ветвистости листьев }\end{array}$ & & & \\
\hline
\end{tabular}

${ }^{*} p<0.05,{ }^{*} p<0.01$. 
Гипотеза 3 подтвердилась, так как число ошибок в тесте Дж. Кагана коррелирует со значениями метрики «Среднее число родителей в узлах», характеризующей запутанность онтологии, что подтверждает данную гипотезу. Кроме того, это число ошибок коррелирует с метриками «Минимальная глубина», «Коэффициент ветвистости семейств» и обратно коррелирует с взвешенным коэффициентом ветвистости листьев.

Наконец, гипотеза 4 также полностью подтвердилась: «широкие» категоризаторы построили онтологии большего размера (по числу концептов), при подробном анализе оказалось, что это было достигнуто за счет большего количества «детей» у каждого родительского концепта. Соответственно, результаты теста «Средние суждения» коррелируют с такими метриками, как «Средняя ширина онтологии», «Количество листьев», «Абсолютная мощность семейств» и т.п. Кроме того, они коррелируют со среднеквадратичным отклонением относительной ширины онтологии. Последнее говорит о том, что количество концептов на соседних уровнях и в различных ветках существенно разнится, а значит, онтологии у широких категоризаторов не сбалансированы.

Несмотря на объективность метрического способа оценки онтологий, у этого способа есть такой существенный недостаток, как формальный подход к онтологиям, отсутствие семантического анализа. Проведя подобный анализ вручную, мы обнаружили, что онтологии, построенные поленезависимыми испытуемыми, имеют более строгую и понятную структуру. Однако зачастую эта стро- гость и простота достигается за счет отбрасывания концептов, не укладывающихся в построенную онтологию; таким образом, полнота онтологии приносится в жертву ее стройности.

Подводя итоги, можно сказать, что хороший онтологист должен предпочтительно обладать такими характеристиками когнитивного стиля, как рефлективность (т.е. наилучшие результаты показали испытуемые, продемонстрировавшие большее латентное время первого ответа и совершившие мало ошибок в тесте Кагана) и узкая категоризация.

Также можно предположить, что при коллективном построении онтологий может оказаться полезным включение в группу широких категоризаторов наряду с поленезависимым участником, чтобы сочетать эффективность первых в генерировании большого числа подклассов и вторых в реструктурировании онтологий. Эта гипотеза была проверена при изучении построения онтологий в группе.

\section{«Коллективная»фаза}

В «коллективной» фазе исследовалась специфика коллективной разработки онтологий как при работе в парах, так и в группах по 3-5 человек. Была сделана попытка определить, каким образом формируется коллективный стиль категоризации.

Построение онтологии в группе может идти по одному из двух вариантов:

1) коллективная разработка с «чистого листа»;

2) разработка единой онтологии на основе использования двух или более заготовок. 
Наибольший практический интерес представляет второй вариант. В этом случае два (или более) студента, выполняющие функции аналитиков, фактически последовательно совершают следующие действия (Choi et al., 2006):

- сопоставление онтологий (ontology mapping) - определение набора пересекающихся концептов, синонимов или уникальных концептов исходных онтологий;

- отображение онтологий (ontology alignment) - установление связей между концептами исходных онтологий;

- слияние онтологий (ontology merging) - создание одной новой согласованной онтологии, основанной на нескольких исходных онтологиях одной области;

- интеграцию онтологий (ontology integration) - создание одной онтологии предметной области на основе исходных онтологий из разных областей.

Все вышеперечисленные действия могут выполняться вручную, полуавтоматически и автоматически. Большинство работ в этой области посвящены автоматическим и полуавтоматическим методам, тогда как ручному объединению онтологий уделено довольно мало внимания.

Эксперименты показали, что слияние происходит по одному из сценариев:

- сценарий компромисса (синтеза): результирующая онтология была мало похожа на своих предшественников, а скорее являлась результатом переосмысления испытуемыми их знаний о предметной области;

- дизъюнктивный сценарий (поглощение): бо́льшая по мощности онтология поглощала меньшую, и в результирующей онтологии происходило объединение вершин одного уровня;

- конъюнктивный сценарий (пересечение): происходило сокращение вершин, и результирующая онтология включала только пересечение вершин одного уровня.

Сопоставление этих сценариев с когнитивными стилями испытуемых выявило следующие закономерности:

- конъюнктивные сценарии предпочитали использовать поленезависимые испытуемые;

- дизъюнктивные сценарии чаще применялись полезависимыми испытуемыми.

Рисунки 6-8 демонстрируют пример слияния онтологий по «компромиссному» сценарию.

\section{Заключение}

Разработка учебных понятийных структур знаний, или онтологий, сегодня является новым трендом в образовании, особенно в «мягких» предметных областях, где определения размыты, теории противоречивы и понятийная база активно изменяется. Учебные онтологии способствуют более глубокому освоению предмета, а также применяются для оценки знаний (Гаврилова и др., 2000; Dall'Alba, Barnacle, 2007; Dicheva, 2008). И преподаватель, и студент при этом могут выступать в двух ролях: аналитика (человека, извлекающего знания) и эксперта (человека, владеющего знаниями) предметной области.

Эксперименты показали, что высокая рефлективность и узкая категоризация является наилучшим 
Пример онтологии, построенной первым испытуемым

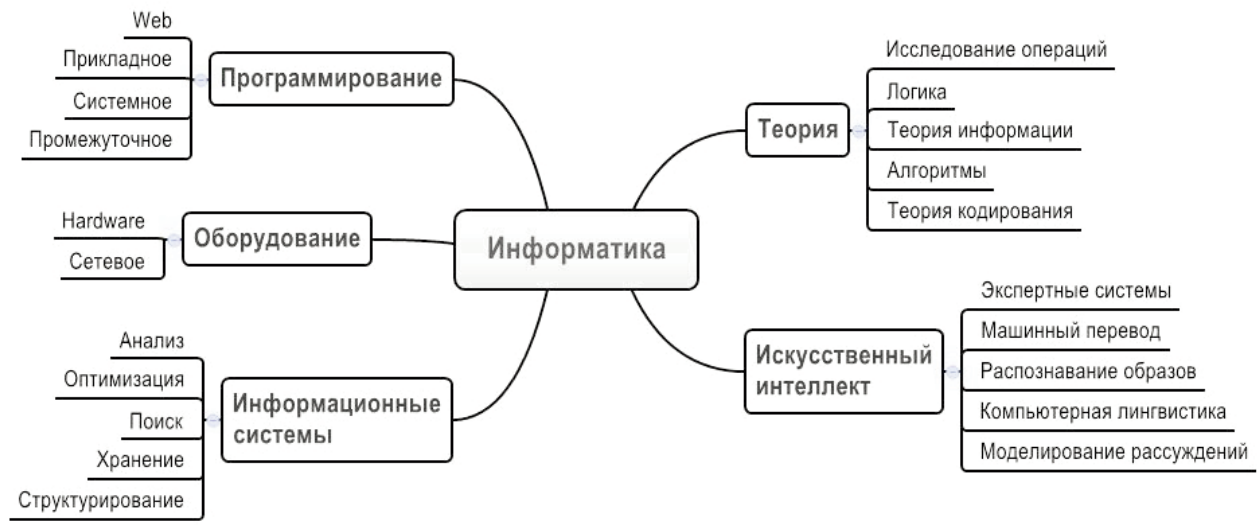

Рисунок 7

Пример онтологии, построенной вторым испытуемым

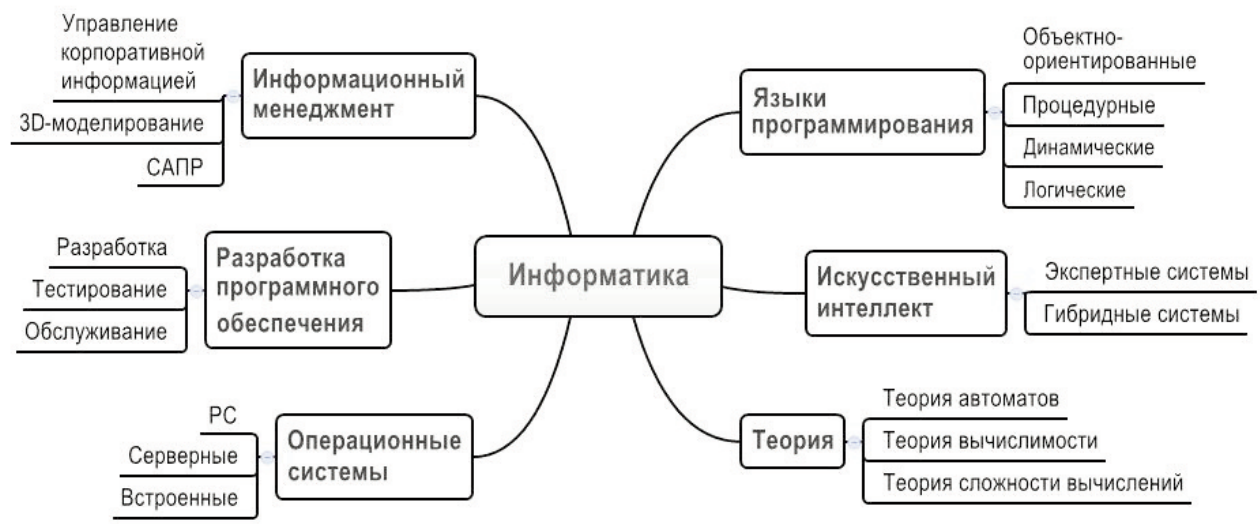

сочетанием характеристик когнитивного стиля испытуемого для структурирования знаний и построения качественных онтологий. Таким образом, можно рекомендовать поручать работу по составлению учебных и научных онтологий обладателям подобных характеристик. Для коллективного построения онтологий имеет смысл рекомендация по включению в группы по разработке онтологий «широких категоризато- ров» наряду с «поленезависимыми» участниками.

Исследование специфики коллективной разработки онтологий при работе в парах и в группах по 3-5 человек позволило выявить предпочтение дизъюнктивных сценариев поленезависимыми участниками и, в свою очередь, конъюнктивных сценариев полезависимыми участниками.

При этом наблюдалась парадоксальная ситуация: если в группу 
Пример онтологии, построенной двумя испытуемыми
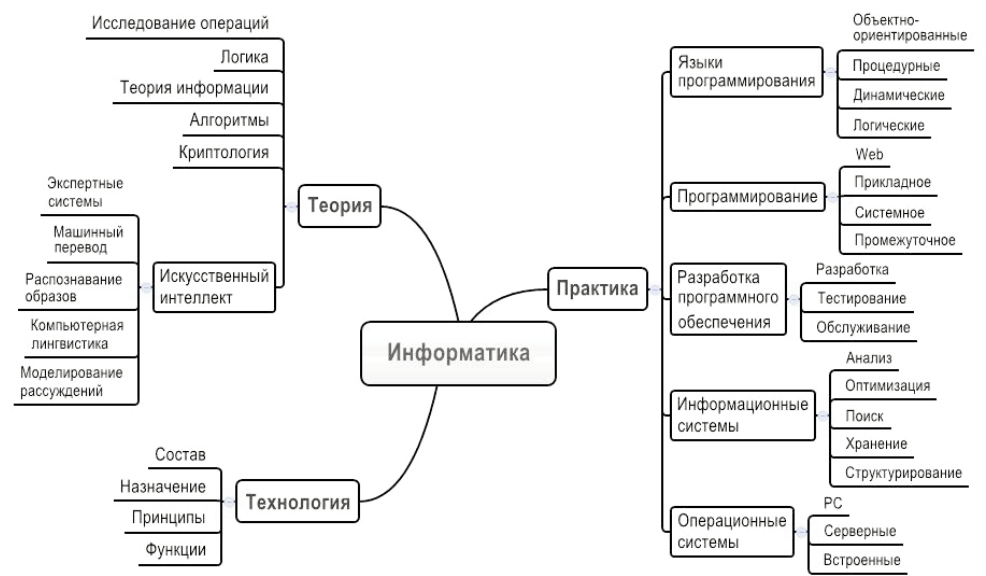

объединялись студенты, чьи индивидуальные онтологии были «хорошие», то общая онтология часто оказывалась намного хуже. Если же в группу попадали авторы разных по качеству онтологий, то им легче удавалось договориться. В итоге структура общей онтологии основывалась на лучшей индивидуальной онтологии в группе, при этом она обогащалась за счет деталей остальных. Смешанные группы не только демонстрировали лучший результат, но и показывали высокую степень эффективности такого способа обучения. У студентов, чьи изначальные онтологии были плохи, при групповой работе было заметно значительное улучшение. Последую- щие их онтологии были намного лучше.

Теоретическая значимость данных результатов высока для дальнейшего применения когнитивной психологии и онтологического инжиниринга в обучении. Практическая значимость работы актуальна не только для сферы образования, но и для создания научных онтологий (Загорулько, 2008; Массель, 2010; Koznov, 2012). Также сегодня групповая разработка онтологий применяется в управленческом консалтинге и других сферах деятельности, связанных с коллективной интеллектуальной творческой работой (Кириков и др., 2014; Кудрявцев и др., 2009; Разин, Тузовский, 2012; Zagoruiko et al., 2007).

\section{Литература}

Аллахвердов, В. М. (2006). Экспериментальная психология познания. Когнитивная логика сознательного и бессознательного. СПб.: Изд-во Санкт-Петербургского университета.

Афанасьев, В. В., Резаков, Р. Г. (2012). Педагогическая диагностика качества познавательной деятельности студентов. Вестник МГПУ. Серия «Педагогика и психология», 3(21), 33-41. 
Болотникова, Е. С., Гаврилова, Т. А., Горовой, В. А. (2011). Об одном методе оценки онтологий. Известия РАН, Теория и системь управления, 3, 98-110.

Бьюзен, Т., Бьюзен, Б. (2014). Супермышление. Минск: Попурри.

Величковский, Б. М. (2006). Когнитивная наука: Основы психологии познания (в 2 т.). М.: Смысл.

Гаврилова, Т. А. (2002). Логико-лингвистическое управление как введение в инженерию знаний. Новости искусственного интеллекта, 6, 28-33.

Гаврилова, Т. А. (2005). Об одном подходе к онтологическому инжинирингу. Новости искусственного интеллекта, 3, 25-31.

Гаврилова, Т. А., Горовой, В. А., Болотникова, Е. С. (2009). Оценка когнитивной эргономичности онтологии на основе анализа графа. Искусственный интеллект и принятие решений, 3, 33-41.

Гаврилова, Т. А., Лещева, И. А., Лещев, Д. В. (2000). Использование онтологий в качестве дидактического средства. Искусственньй интеллект, 3, 34-39.

Гаврилова, Т.А., Лещева, И. А., Страхович, Э.В.(2011). Об использовании визуальных концептуальных моделей в преподавании. Вестник Санкт-Петербургского университета. Серия 8. Менеджент, 4, 124-150.

Гаврилова, Т. А., Муромцев, Д. И. (2007). Интеллектуальные технологии в менеджменте: инструменты и системы: Уиебное пособие. СПб.: Изд-во «Высшая школа менеджмента»; Издат. дом Санкт Петербургского государственного университета.

Загорулько, Ю. А. (2008). Автоматизация сбора онтологической информации об интернетресурсах для портала научных знаний. Известия Томского политехнического университета, 312(5), 114-119.

Кириков, И. А., Колесников, А. В., Румовская, С. Б. (2014). Функциональная гибридная интеллектуальная система для поддержки принятия решений при диагностике артериальной гипертензии. Системь и средства информатики, 24(1), 153-179.

Косихин, В. В. (2012). Психологическое содержание и диагностика когнитивного стиля «Диапазон эквивалентности». Психология. Журнал Высшей школь экономики, 9(2), 116-131.

Котова, Е. Е. (2013). Моделирование индивидуальных программ обучения студентов с учетом личностных параметров когнитивно-стилевого потенциала. Человеческий фактор: проблемъ психологии и эргономики, 4(67), 124-128.

Кубрякова, Е. С., Демьянков, В. З., Панкрац, Ю. Г., Лузина, Л. Г. (1997). Краткий словарь когнитивных терминов. М.: Филологический факультет МГУ им. М. В. Ломоносова.

Кудрявцев, Д. В., Григорьев, Л. Ю., Кислова, В. В., Жулин, А. Б. (2009). Административное моделирование на основе онтологий. Вопросы государственного и мунищипального управления, 1, 157-169.

Лещева, И. А., Котова, Е. Е. (2012). Исследование взаимосвязей между когнитивным стилем эксперта и построенными им онтологиями. В кн. Трудь 1-го Международного симпозиума «Гибридные и синергетические интеллектуальные системы: теория и практика ГИСИС 2012» (с. 232-240). Калининград: Изд-во Балтийского федерального университета им. И. Канта.

Любимов, Л. Л. (2007). Школа и знаниевое общество в России. Вопросы образования, 4, 116-140.

Массель, Л. В. (2010). Применение онтологического, когнитивного и событийного моделирования для анализа чрезвычайных ситуаций в энергетике. Проблемь безопасности $и$ чрезвычайных ситуаций, 2, 34-43.

Разин, В. В., Тузовский, А. Ф. (2012). Метод принятия решений на основе анализа ситуаций и семантических технологий. Известия Томского политехнического университета, 321(5), 188-193. 
Соловьев, В. Д. (2007). Методика анализа семантических репрезентаций: на пути к естественнонаучной парадигме. В кн. Проблема представления (репрезентации) в языке: Типь и форматы знаний (с. 80-85). М.: Институт языкознания РАН.

Солсо, Р. (2006). Когнитивная психология. СПб.: Питер.

Холодная, М. А. (2004). Когнитивные стили. О природе индивидуального ума (2-е изд.). СПб.: Питер.

Холодная, М. А. (2009). Структурно-интегративная методология в исследовании интеллекта. Психологические исследования, Appendix 1(3), 195-204. Режим: http://psystudy.ru/index.php/ num/appendix1-3/66-kholodnaya3a.html

Chernigovskaya, T. (1999). Neurosemiotic approach to cognitive functions. Journal of the International Association for Semiotic Studies - SEMIOTICA, 127(1/4), 227-237.

Choi, N., Song, I.-Y., \& Han, H. (2006). A survey on ontology mapping. ACM SIGMOD Record, 35(3), $34-41$.

Dall'Alba, G., \& Barnacle, R. (2007). An ontological turn for higher education. Studies in Higher Education, 32(6), 679-691.

Dicheva, D. (2008). Ontologies and semantic web for e-learning. In H. H. Adelsberger, Kinshuk, J. M. Pawlowski, \& D. Sampson (Eds.), Handbook on information technologies for education and training (pp. 47-65). Berlin/Heidelberg: Springer.

Fillenbaum, S. (1959). Some stylistic aspects of categorizing behavior. Journal of Personality, 27, 187-195.

Gangemi, A., Catenacci, C., Ciaramita, M., \& Lehmann, J. (2005). Ontology evaluation: A review of methods and an integrated model for the quality diagnostic task. Technical Report. Retrieved from http://www.loa.istc.cnr.it/old/Files/OntoEval4OntoDev_Final.pdf

Gangemi, A., Catenacci, C., Ciaramita, M., \& Lehmann, J. (2006). Modelling ontology evaluation. In Y. Sure \& J. Domingue (Eds.), Lecture Notes in Computer Science: Vol. 4011. The semantic web: research and applications (pp. 140-154). Berlin: Springer. doi: 10.1007/11762256_13

Gruber, T. R. (1993). A translation approach to portable ontology specifications. Knowledge Acquisition, 5(2), 199-220.

Guarino, N., \& Giaretta, P. (1995). Ontologies and knowledge bases. Towards a terminological clarification. In N. Mars (Ed.), Towards very large knowledge bases: Knowledge building and knowledge sharing (pp. 25-32). Amsterdam: IOS Press.

IDC. (2014). The Digital Universe of Opportunities: Rich Data and the Increasing Value of the Internet of Things. EMC Digital Universe study with research and analysis by IDC. Retrieved from http://www.emc.com/leadership/digital-universe/index.htm

Kagan, J. (1966). Reflection-impulsivity: The generality and dynamics of conceptual tempo. Journal of Abnormal Psychology, 71, 17-24.

Koznov, D. V. (2012). Teaching to write software engineering documents with focus on document design by means of mind maps. In V. Uskov (Ed.), Proceedings of the IASTED International Conference on Computers and Advanced Technology in Education, CATE 2012 (pp. 112-118). Napoli, Italy: ACTA Press. doi: 10.2316/P.2012.774-036

Lakoff, G. (1987). Women, fire, and dangerous things: What categories reveal about the mind. Chicago: University of Chicago Press.

Miller, G. (1956). The magical number seven, plus or minus two: Some limits on our capacity for processing information. Psychological Review, 63, 81-97.

Noy, N. F., \& McGuinness, D. L. (2001). Ontology Development 101: A guide to creating your first ontology (Stanford Knowledge Systems Laboratory Technical Report KSL-01-05 and Stanford Medical 
Informatics Technical Report SMI-2001-0880). Retrieved from http://protegewiki.stanford.edu/wiki/Ontology101

Pettigrew, T. F. (1958). The measurement and correlates of category width as a cognitive variable. Journal of Personality, 26, 532-544.

Rosch, E. (1999). Principles of categorization. In E. Margolis \& S. Laurence (Eds.), Concepts: core readings (pp. 189-206). Cambridge, MA: MIT Press.

Thurstone, L. L. (1944). A factorial study of perception. Chicago: University of Chicago Press.

Witkin, H. A. (1971). A manual for the embedded figures tests. Palo Alto, CA: Consulting Psychologists Press.

Witkin, H. A., Moore, C. A., Goodenough, D. R., \& Cox, P. W. (1977). Field-dependent and field-independent cognitive styles and their educational implications. Review of Educational Research, $47(1), 1-64$.

Zagoruiko, N. G., Gulyaevskii, S. E., \& Kovalerchuk, B. Ya. (2007). Ontology of the data mining subject domain. Pattern Recognition and Image Analysis, 17(3), 349-356.
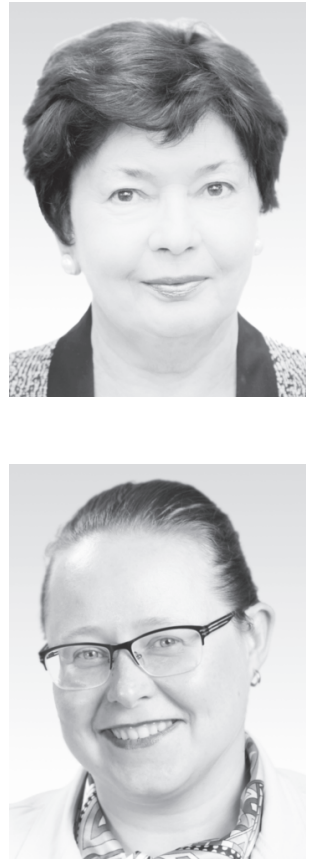

Гаврилова Татьяна Альбертовна - заведующая кафедрой информационных технологий в менеджменте, Высшая школа менеджмента СПбГУ, профессор, доктор технических наук.

Сфера научных интересов: управление знаниями, инженерия знаний, интеллектуальные системы.

Контакты: gavrilova@gsom.pu.ru

Лещева Ирина Анатольевна - старший преподаватель кафедры информационных технологий в менеджменте, Высшая школа менеджмента СПбГУ.

Сфера научных интересов: управление знаниями, онтологии.

Контакты: leshcheva@gsom.pu.ru 


\title{
Conceptual Knowledge Structures and Cognitive Style
}

\author{
Tatiana A. Gavrilova $a^{a}$ Irina A. Leshcheva ${ }^{a}$ \\ ${ }^{a}$ Graduate School of Management (GSOM SPbU), 3 Volkhovskiy Pereulok, St. Petersburg, 199004, \\ Russian Federation
}

\begin{abstract}
Absract
Knowledge structuring is one of the poorly-studied cognitive processes that significantly affect the learning activity processes as well as professional knowledge sharing communications. The paper describes the main results of the study aimed at investigation of the impact of the individual characteristics of cognitive style in the process of information structuring during learning. The stress is put on the study of the cognitive style parameters, which significantly affect the conceptual design features of hierarchical models (the so-called ontologies) that are important for learning and understanding of the material. The main described results are focused on empirical testing of hypotheses about the impact of the individual characteristics of cognitive style (such as field dependence/field independence, category narrowness/width, impulsiveness/ reflexivity) on the individual and collective ontology design features. Some interesting results of the group ontology design work study (in pairs and threesomes) are discussed. They present the relationship between the individual ideas of group members about the domain and the resulting common structure. The sample consists of the Saint-Petersburg Polytechnic University students. The students were tested on the specifics of their individual cognitive styles and after they were asked to create ontologies of the "Computer Science" domain personally and collectively. The obtained results allow to propose some practical recommendations for formation of the efficient groups for collective intellectual work, e.g. data base design, knowledge representation models, business-models and other conceptual structures.
\end{abstract}

Keywords: conceptual structures, ontologies, knowledge engineering, knowledge structuring, categorization, cognitive styles.

\section{References}

Afanasiev, V. V., \& Rezakov, R. G. (2012). Pedagogical diagnostics of quality of students' cognitive activity. Vestnik Moscow City Teacher Training University. Pedagogic and Psychology, 3(21), 33-41.

Allakhverdov, V. M. (2006). Eksperimental'naya psikhologiya poznaniya. Kognitivnaya logika soznatel'nogo $i$ bessoznatel'nogo [Experimental cognitive psychology. The cognitive logic of the conscious and the unconscious]. Saint Petersburg: Saint Petersburg State University Press.

Bolotnikova, E. S., Gavrilova, T. A., \& Gorovoy, V. A. (2011). To a method of evaluating ontologies. Journal of Computer and Systems Sciences International, 50(3), 448-461.

Buzan, T., \& Buzan, B. (2014). Supermyshlenie [Supercognition]. Minsk: Popurri. (Transl. of: Buzan, T., \& Buzan, B. (1996). The mind map book. London: BBC Books).

Chernigovskaya, T. (1999). Neurosemiotic approach to cognitive functions. Journal of the International Association for Semiotic Studies - SEMIOTICA, 127(1/4), 227-237. 
Choi, N., Song, I.-Y., \& Han, H. (2006). A survey on ontology mapping. ACM SIGMOD Record, 35(3), $34-41$.

Dall'Alba, G., \& Barnacle, R. (2007). An ontological turn for higher education. Studies in Higher Education, 32(6), 679-691.

Dicheva, D. (2008). Ontologies and semantic web for e-learning. In H. H. Adelsberger, Kinshuk, J. M. Pawlowski, \& D. Sampson (Eds.), Handbook on information technologies for education and training (pp. 47-65). Berlin/Heidelberg: Springer.

Fillenbaum, S. (1959). Some stylistic aspects of categorizing behavior. Journal of Personality, 27, $187-195$.

Gangemi, A., Catenacci, C., Ciaramita, M., \& Lehmann, J. (2005). Ontology evaluation: A review of methods and an integrated model for the quality diagnostic task. Technical Report. Retrieved from http://www.loa.istc.cnr.it/old/Files/OntoEval4OntoDev_Final.pdf

Gangemi, A., Catenacci, C., Ciaramita, M., \& Lehmann, J. (2006). Modelling ontology evaluation. In Y. Sure \& J. Domingue (Eds.), Lecture Notes in Computer Science: Vol. 4011. The semantic web: research and applications (pp. 140-154). Berlin: Springer. doi: 10.1007/11762256_13

Gavrilova, T. A. (2002). Logiko-lingvisticheskoe upravlenie kak vvedenie v inzheneriyu znanii [Logic and linguistic management as introduction to engineering of knowledge]. Novosti Iskusstvennogo Intellekta, 6, 28-33.

Gavrilova, T. A. (2005). Ob odnom podkhode k ontologicheskomu inzhiniringu [On the one approach to ontological engineering]. Novosti Iskusstvennogo Intellekta, 3, 25-31.

Gavrilova, T. A., Gorovoy, V. A., \& Bolotnikova, E. S. (2009). Otsenka kognitivnoi ergonomichnosti ontologii na osnove analiza grafa [Evaluation of cognitive ergonomicity of ontology on the basis of graph analysis]. Artificial Intelligence and Decision Making, 3, 33-41.

Gavrilova, T. A., \& Khoroshevsky, V. F. (2000). Bazy znanii intellektual'nykh sistem [Knowledge bases of intellectual systems]. Saint Petersburg: Piter.

Gavrilova, T. A., Leshcheva, I. A., \& Leshchev, D. V. (2000). Ispol'zovanie ontologii v kachestve didakticheskogo sredstva. Iskusstvennyi Intellekt, 3, 34-39.

Gavrilova, T.A., Leshcheva, I. A., \& Strakhovich, E. V. (2011). Ob ispol'zovanii vizual'nykh kontseptual'nykh modelei v prepodavanii [On the usage of visual conceptual models in teaching]. Vestnik of Saint Petersburg State University. Series 8. Management, 4, 124-150.

Gruber, T. R. (1993). A translation approach to portable ontology specifications. Knowledge Acquisition, 5(2), 199-220.

Guarino, N., \& Giaretta, P. (1995). Ontologies and knowledge bases. Towards a terminological clarification. In N. Mars (Ed.), Towards very large knowledge bases: Knowledge building and knowledge sharing (pp. 25-32). Amsterdam: IOS Press.

IDC. (2014). The Digital Universe of Opportunities: Rich Data and the Increasing Value of the Internet of Things. EMC Digital Universe study with research and analysis by IDC. Retrieved from http://www.emc.com/leadership/digital-universe/index.htm

Kagan, J. (1966). Reflection-impulsivity: The generality and dynamics of conceptual tempo. Journal of Abnormal Psychology, 71, 17-24.

Kholodnaya, M. A. (2004). Kognitionye stili. O prirode individual'nogo uma [Cognitive styles. On the nature of individual mind] (2nd ed.). Saint Petersburg: Piter.

Kholodnaya, M. A. (2009). Strukturno-integrativnaya metodologiya v issledovanii intellekta [Structural-integrative methodology in the study of intellect]. Psikhologicheskie Issledovaniya, Appendix 1(3), 195-204. Retrieved from http://psystudy.ru/index.php/num/appendix1-3/66kholodnaya3a.html 
Kirikov, I. A., Kolesnikov, A. V., \& Rumovskaya, S. B. (2014). Functional hybrid intelligent decision support system for diagnostics of arterial hypertension. Systems and Means of Informatics, 24(1), 153-179.

Kosikhin, V. V. (2012). Psychological substance and assessment of the equivalence range cognitive style. Psychology. Journal of the Higher School of Economics, 9(2), 116-131.

Kotova, E. E. (2013). Modelirovanie individual'nykh programm obucheniya studentov s uchetom lichnostnykh parametrov kognitivno-stilevogo potentsiala [Modeling of individual programs for students' education with consideration of personal parameters of cognitive style potential]. Chelovecheskii Faktor: Problemy Psikhologii i Ergonomiki, 4(67), 124-128.

Koznov, D. V. (2012). Teaching to write software engineering documents with focus on document design by means of mind maps. In V. Uskov (Ed.), Proceedings of the IASTED International Conference on Computers and Advanced Technology in Education, CATE 2012 (pp. 112-118). Napoli, Italy: ACTA Press. doi: 10.2316/P.2012.774-036

Kubryakova, E. S., Dem'jankov, V. Z., Pankrats, Yu. G., \& Luzina, L. G. (1997). Kratkii slovar' kognitivnykh terminov [A shorter dictionary of cognitive terms]. Moscow: Philological Faculty of Moscow State University.

Kudryavtsev, D. V., Grigoriev, L. Yu,, Kislova, V. V., \& Zhulin, A. B. (2009). Administrativnoe modelirovanie na osnove ontologii [Administrative modeling on the basis of ontology]. Public Administration Issues, 1, 157-169.

Lakoff, G. (1987). Women, fire, and dangerous things: What categories reveal about the mind. Chicago: University of Chicago Press.

Leshcheva, I. A., \& Kotova, E. E. (2012). Issledovanie vzaimosvyazei mezhdu kognitivnym stilem eksperta i postroennymi im ontologiyami [A study of interrelations between cognitive style of an expert and his designed ontologies]. In Trudy 1-go Mezhdunarodnogo simpoziuma "Gibridnye i sinergeticheskie intellektual'nye sistemy: teoriya i praktika GISIS 2012” [Writings of the First International Symposium "Hybrid and synergetic intellectual systems: Theory and practice HISIS 2012”] (pp. 232-240). Kaliningrad: Immanuel Kant Baltic Federal University.

Lyubimov, L. L. (2007). School and knowledge society in Russia. Voprosy obrazovaniya / Educational Studies, 4, 116-140.

Massel, L. V. (2010). Primenenie ontologicheskogo, kognitivnogo i sobytiinogo modelirovaniya dlya analiza chrezvychainykh situatsii v energetike [The application of ontological, cognitive and event modeling for analysis of emergency situations in energy engineering]. Problemy Bezopasnosti $i$ Chrezoychainykh Situatsii, 2, 34-43.

Miller, G. (1956). The magical number seven, plus or minus two: Some limits on our capacity for processing information. Psychological Revier, 63, 81-97.

Noy, N. F., \& McGuinness, D. L. (2001). Ontology Development 101: A guide to creating your first ontology (Stanford Knowledge Systems Laboratory Technical Report KSL-01-05 and Stanford Medical Informatics Technical Report SMI-2001-0880). Retrieved from http://protegewiki.stanford.edu/wiki/Ontology101

Pettigrew, T. F. (1958). The measurement and correlates of category width as a cognitive variable. Journal of Personality, 26, 532-544.

Razin, V. V., \& Tuzovsky, A. F. (2012). The decision making method based on analysis of situations and semantic technologies. Bulletin of the Tomsk Polytechnic University. Geo Assets Engineering, 321(5), 188-193. 
Rosch, E. (1999). Principles of categorization. In E. Margolis \& S. Laurence (Eds.), Concepts: core readings (pp. 189-206). Cambridge, MA: MIT Press.

Solovyev, V. D. (2007). Metodika analiza semanticheskikh reprezentatsii: na puti k estestvennonauchnoi paradigme [The evaluation method of semantic representations: Towards the natural scientific paradigm]. In Problema predstavleniya (reprezentatsii) v yazyke: Tipy i formaty znanii [The problem of representations in language: Types and formats of knowledge] (pp. 80-85). Moscow: Institute of Linguistics of Russian Academy of Sciences.

Solso, R. (2006). Kognitionaya psikhologiya [Cognitive psychology]. Saint Petersburg: Piter. (Transl. of: Solso, R. (1995). Cognitive psychology (4th ed.). Boston: Allyn and Bacon).

Thurstone, L. L. (1944). A factorial study of perception. Chicago: University of Chicago Press.

Velichkovsky, B. M. (2006). Kognitivnaya nauka: Osnozy psikhologii poznaniya [Cognitive science: The fundamentals of cognitive psychology] (in 2 vols.). Moscow: Smysl.

Witkin, H. A. (1971). A manual for the embedded figures tests. Palo Alto, CA: Consulting Psychologists Press.

Witkin, H. A., Moore, C. A., Goodenough, D. R., \& Cox, P. W. (1977). Field-dependent and field-independent cognitive styles and their educational implications. Review of Educational Research, $47(1), 1-64$.

Zagoruiko, N. G., Gulyaevskii, S. E., \& Kovalerchuk, B. Ya. (2007). Ontology of the data mining subject domain. Pattern Recognition and Image Analysis, 17(3), 349-356.

Zagorulko, Yu. A. (2008). Automation of ontologic information gathering about Internet-resources for the scientific knowledge portal. Bulletin of the Tomsk Polytechnic University. Geo Assets Engineering, 312(5), 114-119.

Tatiana A. Gavrilova - head of Information Technologies in Management Department, Graduate School of Management (GSOM SPbU), professor, D.Sc.

Research area: knowledge engineering, knowledge management, ontologies for teaching and management.

E-mail: gavrilova@gsom.pu.ru

Irina A. Leshcheva - senior lecturer, Graduate School of Management (GSOM SPbU)

Research area: knowledge management, knowledge engineering, ontologies.

E-mail: leshcheva@gsom.pu.ru 